\title{
Core-hydrogen-burning RSGs in the early globular clusters
}

\author{
Dorottya Szécsi ${ }^{1}$, Jonathan Mackey ${ }^{2}$ and Norbert Langer ${ }^{1}$ \\ ${ }^{1}$ Argelander-Institut für Astronomie, Auf dem Hügel 71, Bonn-53121, Germany \\ email: dorottya@astro.uni-bonn.de, nlanger@astro.uni-bonn.de
}

${ }^{2}$ I. Physikalisches Institut, Universität zu Köln, Zülpicher Straße 77, 50937 Köln, Germany email: mackey@ph1.uni-koeln.de

\begin{abstract}
The first stellar generation in galactic globular clusters contained massive lowmetallicity stars (Charbonnel et al. 2014). We modelled the evolution of this massive stellar population and found that such stars with masses 100-600 $\mathrm{M}_{\odot}$ evolve into cool RSGs (Szécsi et al. 2015). These RSGs spend not only the core-He-burning phase but even the last few $10^{5}$ years of the core-H-burning phase on the SG branch. Due to the presence of hot massive stars in the cluster at the same time, we show that the RSG wind is trapped into photoionization confined shells (Mackey et al. 2014). We simulated the shell formation around such RSGs and find them to become gravitationally unstable (Szécsi et al. 2016). We propose a scenario in which these shells are responsible for the formation of the second generation low-mass stars in globular clusters with anomalous surface abundances.
\end{abstract}

Keywords. globular clusters: general, stars: abundances, stars: formation, stars: supergiants

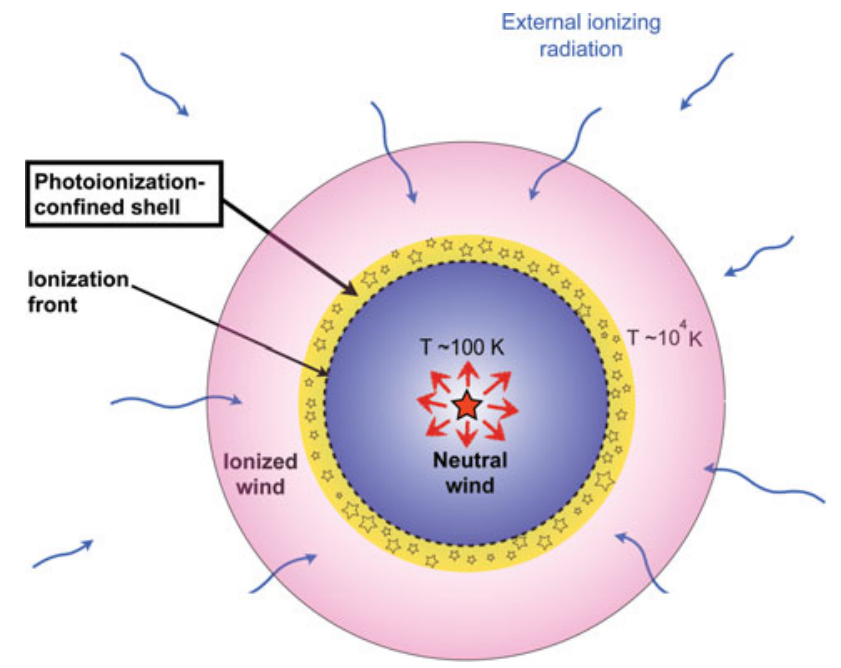

Figure 1. Photoionization-confined shell around a core-hydrogen-burning cool supergiant star (red five-point star symbol). The second generation of low mass stars (open star symbols) are formed in the shell. This scenario could be common in the first few megayears of the early globular clusters, explaining the pollution of the second generation (Szécsi et al. 2016).

\section{References}

Charbonnel, C., Chantereau, W., Krause, M., Primas, F., \& Wang, Y. 2014, A\&6A, 569, L6

Mackey, J., Mohamed, S., Gvaramadze, V. V. et al. 2014, Nature, 512, 282

Szécsi, D., Langer, N., Yoon, S.-C. et al. 2015, A\& $\mathcal{E}$, 581, A15

Szécsi, D., Mackey, J., \& Langer, N. 2016, in preparation 